\title{
Refractory Glioma
}

National Cancer Institute

\section{Source}

National Cancer Institute. Refractory Glioma. NCI Thesaurus. Code C147107.

Glioma that does not respond to treatment. 